\title{
BMJ Open Effect of a combined programme of dietary restriction and physical activity on the physical function and body composition of obese middle-aged and older adults with knee OA (DRPA): protocol for a feasibility study
}

\author{
Asma S Alrushud, ${ }^{1,2}$ Alison B Rushton, ${ }^{1,3}$ Gurjit Bhogal, ${ }^{4}$ Fraser Pressdee, ${ }^{4}$ \\ Carolyn A Greig ${ }^{1,3,5}$
}

To cite: Alrushud AS, Rushton AB, Bhogal G, et al. Effect of a combined programme of dietary restriction and physical activity on the physical function and body composition of obese middleaged and older adults with knee OA (DRPA): protocol for a feasibility study. BMJ Open 2018;8:e021051. doi:10.1136/ bmjopen-2017-021051

- Prepublication history and additional material for this paper are available online. To view these files, please visit the journal online (http://dx.doi. org/10.1136/bmjopen-2017021051).

Received 9 December 2017

Revised 20 June 2018

Accepted 12 September 2018

D Check for updates

(C) Author(s) (or their employer(s)) 2018. Re-use permitted under CC BY-NC. No commercial re-use. See rights and permissions. Published by BMJ.

For numbered affiliations see end of article.

Correspondence to

Asma S Alrushud;

asa314@student.bham.ac.uk

\section{ABSTRACT}

Introduction Knee osteoarthritis $(\mathrm{OA})$ is the most common chronic illness among older adults. Up to the submission date of this protocol, there are no published UK studies reporting the efficacy of a combined intervention programme of physical activity and dietary restriction on the musculoskeletal function of obese older adults with knee $0 \mathrm{~A}$ in spite of the clinical recommendation for exercise and diet for people with knee OA. The aim of this study is to assess the feasibility and acceptability of a combined dietary restriction and physical activity intervention programme and collect preliminary data. Method and analysis This single-arm intervention study is scheduled to begin in September 2017 and conclude in November 2018. It will take place at the Royal Orthopaedic Hospital (ROH), Birmingham and the School of Sport, Exercise and Rehabilitation Sciences (SportExR), University of Birmingham. Participants will receive a physiotherapy usual care programme for knee $0 \mathrm{~A}$ for 1 month, after which they will continue to exercise in their local gym/ leisure facility for 3 months. Participants will also follow dietary restriction throughout the 4-month intervention. Mixed analysis techniques will be used to analyse the quantitative and qualitative outcome measures.

Ethics and dissemination It is approved by ROH R\&D Foundation Trust and the Health Research Authority. The Consort Guidelines and checklist will be reviewed prior to generating any publications for the trial to ensure they meet the standards required for submission to high-quality peer-reviewed journals.

Trial registration number ISRCTN12906938.

\section{INTRODUCTION}

Knee osteoarthritis (OA) is a major public health problem worldwide and a common condition in older adults due to high incidence and prevalence rates. ${ }^{12}$ Approximately $3.64 \%$ of the global population is affected by knee OA. ${ }^{3}$ In 2010, 4.71 million people

\section{Strengths and limitations of this study}

- Mixed-methods data collection to provide detailed information about the combined programme.

- Collaboration with secondary healthcare providers to conduct the study.

- Measurement of outcomes across several domains including biomarkers for knee osteoarthritis to inform the selection of the appropriate outcome measures for a future study.

- Due to limited resources, this will be a single-centre feasibility study.

aged $\geq 45$ years sought treatment for knee OA in England. ${ }^{4}$ The impact of knee OA includes pain, decline in physical function, reduced quality of life (QOL) and increased disability. ${ }^{5}$

In addition to advancing age, obesity is also a known risk factor for developing $\mathrm{OA}$ and causing disability. ${ }^{6-10}$ Obesity increases the negative impact of the disease and patients with arthritis may need surgical intervention to reduce body weight and thereby relieve pain. $^{7}$

In overweight and obese adults, knee OA management should focus mainly on weight reduction. ${ }^{11}{ }^{12}$ The evidence supports a calorie-restricted diet in adults but the optimal $\operatorname{method}(\mathrm{s})$ for weight reduction are still varied in terms of intervention length. Introducing a low-calorie diet is challenging in older adults with knee OA because of the need to maintain the appropriate intake of the important nutrients. For example, maintenance of the recommended daily calcium intake is essential for women who may be at risk of osteoporosis. ${ }^{13}$ For overweight and obese patients with knee OA, previous studies 
have indicated that the ultimate goal of weight loss should be at least $10 \%$ of body weight, in order to provide significant pain reduction. ${ }^{1415}$ Recently, a systematic review (SR) recommended that total body weight should be reduced by at least $5 \%$ over a period of 20 weeks in this population to experience symptoms improvement. ${ }^{15}$

The European League Against Rheumatism and the Osteoarthritis Research Society International (OARSI) guidelines recommend exercise as a core intervention for knee OA management. ${ }^{16}{ }^{17}$ Patients with mild to moderate knee OA have been shown to benefit from muscle strengthening and aerobic exercises with respect to decreasing pain and improving mobility. ${ }^{18}$ In the UK, usual care is considered as the first line of knee OA treatment in clinical practice ${ }^{19}$ and its main foci are exercise and advice. ${ }^{20} 21$ Exercise intervention may include different types of exercise, for example, strengthening exercise (weight bearing and non-weight bearing), range of motion (ROM) and stretching exercise. Advice may concentrate on the importance of exercise and provide patients with information about pain relief strategies, for instance, analgesics and home heat therapy. ${ }^{20}{ }^{21}$ However, the most effective way of delivering exercise to optimise patient outcome is still unclear, particularly in older people with knee OA. ${ }^{21}$ The published literature reports that both aerobic walking and quadriceps strengthening exercises have a positive effect on participants with knee $\mathrm{OA}$ and, overall, there is no superiority of one approach over the other. ${ }^{22}$

Combining weight loss and exercise regimens is recommended for older adult patients with knee OA. ${ }^{23-26} \mathrm{~A}$ recent SR investigated the effectiveness of combining physical activity and dietary restriction interventions on the musculoskeletal function of overweight and obese older adults with knee OA. ${ }^{27}$ Only one pilot study ${ }^{23}$ and two trials, the Arthritis, Diet, and Activity Promotion Trial $^{24}$ and the Intensive Diet and Exercise for Arthritis ${ }^{25}$ were identified. The reviewers concluded that there was unclear quality of evidence for the benefit of combining interventions. ${ }^{27}$ To date of developing this protocol, there are no published UK studies reporting efficacy of a combined intervention programme for older adults. As a consequence, there is a need to develop a study incorporating a mixed methods approach to investigate the acceptability, feasibility as well as the efficacy of a combined intervention programme in older adults with knee OA.

The aim of this feasibility study is to assess the feasibility, acceptability and collect preliminary data to inform the design of a definitive trial incorporating a combined intervention programme.

\section{METHODS AND ANALYSIS}

\section{Research questions and objectives}

This 4-month intervention study aims to answer the central question 'Will the combined intervention programme be feasible and acceptable for the participants and the Royal Orthopaedic Hospital (ROH) physiotherapy staff?'

Additional research questions are formulated:

1. What is the potential for efficacy of the proposed combined intervention programme, as indicated by preliminary data evaluating improvement in outcome measures including: Western Ontario and McMaster Universities Osteoarthritis Index (WOMAC), body weight, waist circumference (WC), body mass index (BMI), musculoskeletal function including; knee ROM and muscle power, musculoskeletal function including; stair climb and timed up-and-go, pain, QOL and markers of joint remodelling?

2. Are there any associations between changes in body weight, knee pain, BMI and markers of joint remodelling?

Primary objective: Will the combined intervention programme be feasible and acceptable, for the targeted population and the $\mathrm{ROH}$ physiotherapy department staff?

Secondary objectives:

1. Assess suitability of main trial measures.

2. Investigate associations between changes in body weight, knee pain, BMI and markers of joint remodelling.

It is hypothesised that the combined programme will be feasible and acceptable either for the targeted population or the ROH physiotherapy department or both. All outcome measures will be assessed for suitability. After 4 months of intervention, it is hypothesised that losing weight will be associated with improvement of knee pain and markers of joint remodelling.

\section{Study design}

This is a single-centre feasibility study, consisting of one arm (combined programme of dietary restriction and physical activity) with an embedded qualitative component (focus group for participants and questionnaire for physiotherapy staff).

\section{Study setting}

The combined programme intervention and the physiotherapy staff questionnaire will take place within the $\mathrm{ROH}$, Birmingham. Participant assessments and focus group sessions will be conducted at the University of Birmingham (UoB), UK.

\section{Participants}

We will recruit 30 older adults (men and women aged $\geq 45-90$ years) with BMI $\geq 30 \mathrm{~kg} / \mathrm{m}^{2}$. They will have a diagnosis of knee OA (with or without radiographic evidence) and will not be participating in regular exercise more than twice a week. They will be able to understand verbal and written English and be willing to participate and give informed consent for the study. They will be excluded if they have any significant comorbid diseases that would pose a safety threat or impair ability to participate such as coronary artery disease, severe hypertension, peripheral vascular disease, stroke, congestive heart 
failure, chronic obstructive pulmonary disease, insulin-dependent diabetes, psychiatric disease, renal disease, liver disease, active cancer other than skin cancer, advanced osteoporosis and anaemia or have had previous acute knee injury (moderate-severe). Additional exclusion criteria are resting systolic blood pressure greater than $200 \mathrm{~mm} \mathrm{Hg}$ and resting diastolic blood pressure greater than $100 \mathrm{~mm} \mathrm{Hg}$, neuromuscular impairments that preclude participating in physical activity, visual, hearing or moderate/severecognitive impairments as well as unwillingness to modify diet or physical activity patterns or inability to comply with the intervention because of inability to access a gym/localleisure facility, food allergies or reactions to the calorie-restricted diet.

\section{Intervention}

The intervention will consist of a single education session, physical activity and dietary restriction components. ${ }^{28-31}$

\section{Educational session}

The first physical activity class will be preceded by an educational session lasting for $60 \mathrm{~min}$ introduced by a qualified physiotherapist. Information about causes and management of knee OA, importance of exercise and diet and safety considerations will be given as part of physiotherapy usual care for patients with knee OA. Additional information about dietary restriction will be introduced by a clinical dietician in the last $10-15 \mathrm{~min}$ of the session. Information in this session is based on the principles of self-management theory, ${ }^{32-34}$ self-efficacy theory $^{35}$ and the framework of behaviour change ${ }^{36}$ and addresses changing lifestyle and coping with pain, stiffness and limited activities of daily living. Participants will have the opportunity to ask any further questions.

\section{Physical activity}

Participants will receive a physiotherapy usual care programme for knee OA (moderate intensity stretching and strengthening exercise) in the physiotherapy gym under the supervision of a physiotherapy technician for 1 month (60 min per week); it is based on social cognitive theory and group dynamics. ${ }^{37}$ Participants will begin with a warm-up exercise for $5 \mathrm{~min}$ around the gym including quadriceps, hamstring and gastrocnemius stretches. The group will split into two small groups working across several work stations on ROM, strength, cardio, gait stations for a further $40 \mathrm{~min}$, for example, cycle ergometer, treadmill, leg press, theraband, inner range quads with band gait work using light hand weights. Sit to stand with medicine ball, marching on a trampette, lunges and extension stretches on step, step overs, tandem walking (eyes open-eyes closed), straight leg raising with weights, hamstring curls with weights, bridging, posterior gluteus medius with band, slight hip abduction and through range quads over the edge of a plinth. Group balance work will be carried out towards the end of the session. Cool down will be completed with stretches. All stations will run for approximately 2 min with additional weights and resistance to be added for progression. All participants will receive a home exercise programme as part of their usual care. Home exercise is based on the National Institute of Health and Care Excellence Guidelines for knee $\mathrm{OA}^{38}$ The classes are group based as opposed to individualised. The participants will split into two small groups working across several work stations and will spend approximately $2 \mathrm{~min}$ at each station. Each participant will exercise according to his/her ability and progression will be tailored accordingly. Personalisation is based around the individual participants hobbies/interests and Activities of Daily Livings (ADLs) with advice being offered accordingly. Participants will monitor their own symptom levels and modify intensity accordingly and work within their own defined tolerance levels. After 1 month, participants will continue to exercise in their local gyms/ leisure facilities for a further 3 months. The study team will assist participants with application for a free (or discounted through funding by the study) gym pass via the Birmingham Be Active scheme. Participants will be provided with a programme of recommended exercises to continue in this setting. The participants will be contacted weekly by telephone to encourage adherence. The first 4 weeks will be the adoption stage where the participants will be educated and supported to change their behaviours and the remaining 12 weeks of the study will be for maintenance. ${ }^{35}$

\section{Dietary restriction}

Participants will complete a 3-day food diary and then follow dietary restriction throughout the 4 months of the intervention, aiming for a decrease of 300-500 kcal/ day or 500-1000 kcal/day as appropriate to initial BMI. The diet will be planned and modified (if required) by a clinical dietician. Participants will monitor themselves by completing a daily log.

\section{Outcome measures}

\section{Qualitative component (feasibility objective)}

Feasibility of the combined intervention programme will be measured using a post intervention focus group for participants and a questionnaire for the involved physiotherapy staff. The feasibility outcomes will be based on the categories reported in Bowden et $a l^{39}$ and listed in table 1 .

\section{Focus group}

Three focus groups will be conducted at the end of the intervention to explore the participants' knowledge, experience and views about the combined intervention programme. ${ }^{39-43}$ The focus group will be audiotaped then analysed. A focus group will be conducted for each group $(n=10)$ at the UoB. The chief investigator (CI) (ASA) will lead the discussion by outlining the purpose of the session and discussing consent and confidentiality. Participants' engagement will be encouraged by asking introductory questions about their previous treatment options for knee OA. An example of the participants' focus group 
Table 1 Feasibility measurement categories

\begin{tabular}{|c|c|c|}
\hline Categories & For participants & For physiotherapy staff \\
\hline 1. Efficacy & Do they report benefit? & Do they notice improvement? \\
\hline 2. Adherence & Using sheet for recording their gym sessions & Record participant attendance \\
\hline 4. Acceptability & $\begin{array}{l}\text { Satisfaction with programme and outcomes } \\
\text { Perceived appropriateness } \\
\text { - Expressed interest }\end{array}$ & $\begin{array}{l}\text { Satisfaction with participants' outcomes } \\
\text { Intent to use again } \\
\text { Fit within hospital culture } \\
\text { Perceived positive or negative effects on their } \\
\text { time, gym availability }\end{array}$ \\
\hline $\begin{array}{l}\text { 5. Implementation } \\
\text { and practicality }\end{array}$ & $\begin{array}{l}\text { Degree of execution, success or failure of } \\
\text { execution } \\
\text { Positive/negative effects on target participants } \\
\text { Ability of participants to carry out intervention } \\
\text { activities and cost }\end{array}$ & $\begin{array}{l}\text { Amount, type of resources needed to implement } \\
\text { Factors affecting implementation ease or } \\
\text { difficulty, efficiency, speed or quality of } \\
\text { implementation }\end{array}$ \\
\hline
\end{tabular}

ROH, Royal Orthopaedic Hospital.

discussion guide is presented in online supplementary 1. This topic guide will inform exploration of the participants' experiences of the combined intervention. An experienced researcher (ABR, coinvestigator) will be responsible for audiotaping and observing the session, taking notes and overseeing data analyses.

\section{Questionnaire}

A mixed questionnaire consisting of open and closed questions has been designed to assess the feasibility of the combined intervention programme. ${ }^{39} 42$ The questionnaire consists of 15 items and its design is based on the categories of feasibility (table 1).

\section{Quantitative measures (efficacy objective)}

Preliminary data informing potential for efficacy of the combined intervention programme will be collected using:

1. Physical function (WOMAC) scale.

2. Body weight, WC and BMI.

3. Body composition (body impedance analysis).

4. Musculoskeletal function (knee ROM, lower limb muscle power).

5. Physical performance (stair climb, timed up-and-go).

6. Pain intensity (WOMAC pain subscale).

7. QOL (Short Form-36).

8. Blood markers of joint remodelling, including: Adipokines (serum adiponectin and leptin) and for bone turnover; Bone formation (Procollagen I C-Terminal Propeptide (PICP)); Bone (type I collagen) degradation (cross linked C-telopeptide of Type 1 collagen (CTX-1)); Cartilage formation: type IIA collagen N-propeptide (PIIANP), Cartilage (type II collagen) degradation: fragments of cartilage oligomeric matrix protein (COMP).$^{44}$

These outcome measures were selected based on a previously published $\mathrm{SR}^{27}$ and OARSI recommendation of performance-based tests to assess physical function of people with knee $\mathrm{OA}^{45}$ with consideration of the study aims and practical considerations. Physical function WOMAC scale is the planned primary outcome for the definitive study. ${ }^{46}$

\section{Sample size}

A formal sample size calculation is not appropriate as one of the study outcomes is to collect data which will inform estimates of SDs for use in a sample size calculation for a future definitive trial. The sample size of 30 participants is based on previous reviews of the literature. ${ }^{478}$ To ensure a suitably reliable estimate of SDs (to power a future study), sample sizes between 24 and 50 have been recommended. ${ }^{4950}$ This sample size is also recommended by The National Institute for Health Research. ${ }^{51}$

\section{Procedure}

Participants will be recruited via the ROH outpatient clinic and the physiotherapy department. Potential participants will be identified by a $\mathrm{ROH}$ clinician or senior physiotherapists according to the eligibility criteria. Eligible participants will be provided with a copy of the participant information sheet and a response slip, which will include a telephone number and email address to contact the study team directly and a stamped addressed envelope for those who prefer to respond by post. In 
the case of participants who have given blood recently (within the last 3 months), their general practitioners will be conducted by the study team to provide the results. Participants will be invited to attend the School of Sport, Exercise and Rehabilitation Sciences (SportExR), UoB, where the study procedures will be explained in further detail. Participants will be given the opportunity to ask questions and informed consent will be obtained. An approved copy of the participants informed consent form is presented in online supplementary 2. Baseline data will be collected by the study team. Blinded outcome assessment will not be applicable in this study but will be applied in the definitive trial. Participants will be asked to complete a 3-day food diary (weekday and weekend) and to return it by post to a member of the study team (using the stamped addressed envelope provided to them), and a date will be scheduled to begin the exercise classes at $\mathrm{ROH}$. The first class will be preceded by an educational session about knee OA, during which participants will be given the opportunity to ask any further questions. See figure 1 for the DRPA trial flow diagram. ${ }^{52}$

\section{Patient and public involvement}

The design of this study has been informed by data gained from several focus groups of older people $\geq 55$ years with knee OA at the Control of Movement and Active Ageing Open Day on 22 June 2016 in the SportExR, UoB.

Recruitment

$N=30$ eligible participants will be identified and recruited as 3 groups ( $n=10$ each). They will be provided with a participation information sheet and willing participants will be contacted by the $\mathrm{Cl}$ to join the assessment session

(Week 0) Assessment session at SportExR

- Consent will be taken by $\mathrm{Cl}$ or delegate within the study team

- Outcomes: body weight, height, BMI, waist circumference, body composition, WOMAC, knee ROM, lower limb muscle power, stair climb time, timed up and go, pain intensity and quality of life

- Blood sample

- 3 day food diary to take away

Intervention

(Week 1, 2, 3 \& 4) Exercise (60 min/week) plus diet

Follow-up

(Weeks 5-16) Continuous diet and exercise at local gym (60 min/week)

Participants will be contacted weekly by telephone

Assessment

(Week 4) ( $2^{\text {nd }}$ assessment point) all outcome measures will be recorded except the serum markers and body composition (Week 16) ( $3^{\text {rd }}$ assessment point) all outcome measures will be recorded plus focus group (participants) at SportExR and questionnaire (PT staff) at $\mathrm{ROH}$

Note: Number of participants ( $\mathrm{n}=10$ in 3 consecutive groups)

4 weeks at PT gym and 12 weeks at local gym, total $=16$ weeks of intervention for each group

The expected start date is September 2017

Figure 1 Flowchart of Dietary Restriction and Physical Activity (DRPA) trial flow diagram. BMI, body mass index; Cl, chief investigator; PT, physiotherapy; ROM, range of motion; ROH, Royal Orthopaedic Hospital; SportExR, School of Sport, Exercise and Rehabilitation Sciences; WOMAC, Western Ontario and McMaster Universities Osteoarthritis Index. 
Table 2 Summary of statistical tests

\begin{tabular}{|c|c|c|c|}
\hline Variable & Form of analysis & How it will be reported & Test \\
\hline Age (year) & Continuous & $M(S D)$ & Means and SD \\
\hline Gender & Categorical & Frequency and percentage & Frequency \\
\hline Height (m) & Continuous & $M(S D)$ & Means and SD \\
\hline Weight (kg) & Continuous & $M(S D)$ & Repeated measures ANOVA \\
\hline $\mathrm{BMI}\left(\mathrm{kg} / \mathrm{m}^{2}\right)$ & Continuous & $M(S D)$ & Repeated measures ANOVA \\
\hline WC (m) & Continuous & $M(S D)$ & Repeated measures ANOVA \\
\hline WOMAC & Continuous & $M(S D)$ & Repeated measures ANOVA \\
\hline Knee ROM (degree) & Continuous & $\mathrm{M}(\mathrm{SD})$ & Repeated measures ANOVA \\
\hline Lower limb muscle power & Continuous & $M(S D)$ & Repeated measures ANOVA \\
\hline $\begin{array}{l}\text { Physical function (stair climb and timed up-and- } \\
\text { go) }\end{array}$ & Continuous & $M(S D)$ & Repeated measures ANOVA \\
\hline Pain intensity (WOMAC) & Continuous & $M(S D)$ & Repeated measures ANOVA \\
\hline Quality of life (SF-36) & Continuous & $M(S D)$ & Repeated measures ANOVA \\
\hline Body composition & Continuous & $M(S D)$ & Paired t-tests \\
\hline Markers of joint remodelling & Continuous & $M(S D)$ & Paired t-tests \\
\hline $\begin{array}{l}\text { Associations between changes in body weight, } \\
\text { knee pain, BMI and markers of joint remodelling }\end{array}$ & Continuous & r & Pearson's correlation \\
\hline
\end{tabular}

ANOVA, analysis of variance; BMI, body mass index; WC, waist circumference; WOMAC, Western Ontario and McMaster Universities Osteoarthritis Index; ROM, range of motion; SF-36, short form-36.

\section{Dissemination}

Findings will be presented to members of the public through events held for the purpose of communicating research into ageing, such as the AgeWell event organised for members of the Birmingham 1000 Elders database.

\section{Data analysis plan}

Qualitative analysis techniques will be used to address the feasibility objective

The guidelines provided by Kitzinger ${ }^{40}$ will be followed to conduct the analysis of data obtained from the focus groups. The phenomenology theoretical orientation ${ }^{53-55}$ will use the Krueger ${ }^{56}$ and Ritchie and Spencer $^{57}$ framework analyses. Open questions from the questionnaire will be analysed similarly.

Quantitative analysis will be used to address the efficacy objective All data will be entered into a database and analysed using SPSS statistics for Windows, V.21.0. Armonk, New York, USA: IBM Corp. Baseline characteristics will be descriptively summarised using frequencies, means and SD or median and IQRs, depending on the frequency distribution of the data. Baseline characteristics will be compared with postintervention data using SPSS, paired t-tests or repeated measures analysis of variance for interval or normal variables. All statistical tests will be conducted two-sided with an alpha level of 0.05 . In addition, closed questions from the questionnaire will be analysed using frequencies and percentages. Details of the selected statistical tests are presented in table 2.

Success criteria will be used to determine the feasibility of the combined intervention programme based on the qualitative and quantitative outcomes. ${ }^{58-60}$ The success criteria are presented in table 3 .

\section{Data collection, management and monitoring}

Personal data recorded on all documents will be regarded as strictly confidential and will be handled and stored in accordance with the Data Protection Act 2018.

Personal addresses, email addresses and telephone numbers will be used to contact participants. Details will previously have been provided by individuals for this purpose, and all details will be kept securely. There will be no electronic storage of personal information, emails will be printed and then deleted. All paper data will be stored securely in a locked drawer. Personal data will be kept for 12 months after the end of the study to allow patient contact for dissemination of results.

Participants will always be identified using only their unique trial identification number on the case report form and correspondence between the participating sites. The investigator will maintain documents in strict confidence. In the case of specific issues and/or queries from the regulatory authorities, it will be necessary to have access to the complete trial records, provided that participant confidentiality is protected.

\section{Procedure(s) to account for missing or spurious data}

Individual data sets will be checked by the CI at regular intervals and any discrepancies highlighted and listed. All missing and ambiguous data will be queried. These will be viewed and discussed by the supervisory team. 
Table 3 Success criteria of the combined intervention programme according to the feasibility categories, using the participants and physiotherapy department staff outcomes

\begin{tabular}{|c|c|c|c|}
\hline Feasibility categories & $\begin{array}{l}\text { Participants quantitative } \\
\text { outcomes }\end{array}$ & $\begin{array}{l}\text { Participants qualitative } \\
\text { outcomes }\end{array}$ & Physiotherapy questionnaire \\
\hline & $\begin{array}{l}\text { The combined intervention } \\
\text { programme will be } \\
\text { considered feasible if: }\end{array}$ & $\begin{array}{l}\text { The combined } \\
\text { intervention programme } \\
\text { will be considered } \\
\text { feasible if: }\end{array}$ & $\begin{array}{l}\text { The combined intervention programme will } \\
\text { be considered feasible if there is consensus } \\
\text { across the PT department staff to support } \\
\text { the following points: }\end{array}$ \\
\hline 1. Efficacy & $\begin{array}{l}\text { Significant changes } p<0.05 \\
\text { on the planned } \\
\text { primary outcome for the } \\
\text { definitive study } \\
\text { (WOMAC scale) from } \\
\text { baseline to } 4 \text { months } \\
\text { intervention }^{30}\end{array}$ & & $\begin{array}{l}\text { When they notice any improvement in the } \\
\text { participants outcomes and think that the } \\
\text { combined intervention programme is better } \\
\text { than usual care }\end{array}$ \\
\hline 2. Adherence & $\begin{array}{l}>80 \% \text { in exercise } \\
\text { compliance } \\
>80 \% \text { in dietary } \\
\text { compliance }\end{array}$ & & $\begin{array}{l}\text { Adherence to the classes is similar } \\
\text { compared with usual care }\end{array}$ \\
\hline 4. Acceptability & & $\begin{array}{l}\text { Participants report } \\
\text { (during focus group } \\
\text { discussion) that they } \\
\text { are satisfied and the } \\
\text { intervention programme } \\
\text { is fitted with their } \\
\text { activities of daily living }\end{array}$ & $\begin{array}{l}\text { They are satisfied with participants } \\
\text { outcomes } \\
\text { They would support the use of the combined } \\
\text { programme } \\
\text { The combined intervention programme fits } \\
\text { within the hospital culture } \\
\text { Delivering the combined intervention } \\
\text { programme has not increased their work } \\
\text { load }\end{array}$ \\
\hline
\end{tabular}

PT, Physiotherapy; WOMAC, Western Ontario and McMaster Universities Osteoarthritis Index; SF-36, Short Form-36; ROH, Royal Orthopaedic Hospital.

\section{Storage and analysis of samples}

Two blood samples $(10 \mathrm{~mL}$ each) will be taken from each participant, one at baseline and one after completing 16 weeks of intervention to analyse the biomarkers of joint remodelling. If the participant has not given blood in the last 3 months, an extra sample will be taken during the first visit $(20 \mathrm{~mL})$ for safety testing. Blood samples for storage will be collected, coded and stored in SportExR for 6-12 months. They will be analysed in the Institute of Inflammation and Ageing, UoB under the supervision of a member of the study team. Collection, analysis, storage and destruction of blood samples will be according to local policy and standard operating procedures aligned to the UoB Quality Management System. Any safety bloods (for those participants who may not have had a blood test within the past 3 months) will be taken from SportExR directly to the laboratories of Queen Elizabeth Hospital, Birmingham.

\section{Monitoring and auditing}

This study team will allow monitoring of the study, including access to source documents as requested. The UoB Clinical Research Compliance Team will review at intervals agreed with CI.

\section{ETHICS AND DISSEMINATION}

This study was approved. 
All changes are documented in the trial site file and communicated with the Research Ethics Committee (REC), lead site, sponsor and funder. As stated in the procedure section, eligible participants will be provided with a participant information sheet and their informed consent form will be given at SportExR. Also, the participation information sheet will be given to the physiotherapy staff and their consent will be taken before completing the questionnaire.

The Consort Guidelines and checklist will be reviewed prior to generating any publications for the trial to ensure they meet the standards required for submission to high-quality peer-reviewed journals. The CI owns the data arising from the trial and will analyse, tabulate and prepare a final study report with guidance from the supervisory team. The full study report can be accessed 6 months after completing the trial.

\section{DISCUSSION}

The feasibility of the combined intervention programme will be assessed based on several areas of focus. ${ }^{28}$ It will be considered feasible if the success criteria in table 3 are met. ${ }^{3059}{ }^{60}$ If the participants suggest major adaption to the study design, for example, cancelling the education session, the programme will be considered as unfeasible.

This feasibility study will answer the central question of whether a combined intervention programme is feasible and acceptable. It is hypothesised that a combined intervention programme of dietary restriction plus usual care for obese older adults with knee OA is feasible and acceptable to the ROH physiotherapists. Participants will experience benefits and the therapists will support the usual care programme plus weight loss in this specific sample. All outcome measures including WOMAC, body weight, WC, BMI, musculoskeletal function (knee ROM and muscle power), musculoskeletal function (stair climb and timed up-and-go), pain, QOL and markers of joint remodelling will show evidence of improvement due to reduction in the participants' body weight, inflammation and pain that which lead to overall improvement in function. It is hypothesised that there will be associations between changes in body weight, knee pain, BMI and markers of joint remodelling.

Participants may find that following designed plans of diet more useful than following general advice. This study has based on several factors to improve compliance with the exercise in the remaining 12 weeks of the intervention such as knowledge, providing facility to local gym and weekly phone call. ${ }^{61}{ }^{62}$ The laboratory test will provide exploratory data for new biomarkers in knee OA. However, the results of this study will add clinical evidence to knee OA management in older adults with obesity and it may inform the provision of usual care of knee OA.

The qualitative component of the study will allow the participants to express their views and impressions about the combined intervention programme and this will inform about the optimal body weight reduction methods and the best way of delivering exercise to achieve better outcomes. Also, the views of physiotherapy staff will be invaluable to confirm whether a combined intervention programme of dietary restriction and usual physiotherapy care is feasible in a hospital setting and could be incorporated into usual care.

\section{Amendment}

If the sponsor wishes to make a substantial amendment to the REC application or the supporting documents, the sponsor will submit a valid notice of amendment to the REC for consideration.

\section{Author affiliations}

${ }^{1}$ School of Sport, Exercise and Rehabilitation Sciences, University of Birmingham, Birmingham, UK

${ }^{2}$ College of Applied Medical Sciences, King Saud University, Riyadh, Saudi Arabia ${ }^{3}$ MRC-Arthritis Research UK Centre for Musculoskeletal Ageing and Health, University of Birmingham, Birmingham, UK

${ }^{4}$ The Royal Orthopaedic Hospital NHS Foundation Trust, Birmingham, UK ${ }^{5}$ National Institute for Health Research (NIHR) Birmingham Biomedical Research Centre, Birmingham, UK

Acknowledgements The authors would like to acknowledge the contribution to the protocol design by participants of focus group sessions which were conducted at the SportExR, UoB (22 June 2016). Also, they would like to acknowledge their collaboration with Dr Simon Jones PhD and Conor Bentley RCD, UoB.

Contributors ASA prepared the first draft of the manuscript that has been reviewed and approved by CAG before circulating it to all coauthors. ABR, GB and FP had critically revised the manuscript and provided their input before submission for publication. All the coauthors have been involved in the study design and developing the protocol.

Funding This work is supported by the UoB which is part of a $\mathrm{PhD}$ studentship award to Ms Alrushud PhD who is funded by the Saudi Arabian Cultural Bureau, London and King Saud University, Saudi Arabia.

Competing interests None declared.

Patient consent Obtained.

Ethics approval The study is approved by the West Midlands-Solihull Research Ethics Committee, REC ref: 17/WM/0122, protocol number ERN_16-1432; RG_17024, and V.6.0 approved on 10 January 2018 by HRA and the ROH R\&D Foundation Trust.

Provenance and peer review Not commissioned; externally peer reviewed.

Author note The protocol was developed by the $\mathrm{Cl}$ and her supervisor and reviewed by the coinvestigators which include qualified medical staff at $\mathrm{ROH}$. This is a low-risk study which has received peer review from the student supervisors plus independent peer review by ROH R\&D Dept and an academic colleague (Dr Leigh Breen, SportExR).

Open access This is an open access article distributed in accordance with the Creative Commons Attribution Non Commercial (CC BY-NC 4.0) license, which permits others to distribute, remix, adapt, build upon this work non-commercially, and license their derivative works on different terms, provided the original work is properly cited, appropriate credit is given, any changes made indicated, and the use is non-commercial. See: http://creativecommons.org/licenses/by-nc/4.0/.

\section{REFERENCES}

1. Hootman JM, Helmick CG. Projections of US prevalence of arthritis and associated activity limitations. Arthritis Rheum 2006;54:226-9.

2. Saraboon Y, Aree-Ue S, Maruo SJ. The Effect of Multifactorial Intervention Programs on Health Behavior and Symptom Control Among Community-Dwelling Overweight Older Adults With Knee Osteoarthritis. Orthop Nurs 2015;34:296-308.

3. Vos T, Flaxman AD, Naghavi M, et al. Years lived with disability (YLDs) for 1160 sequelae of 289 diseases and injuries 1990-2010: 
a systematic analysis for the Global Burden of Disease Study 2010. Lancet 2012;380:2163-96.

4. Arthritis Research UK. Osteoarthritis in general practice, data and perspectives (online). $2013 \mathrm{http}: / / \mathrm{www}$.arthritisresearchuk. org/policyand-public-affairs/reports-and-resources/reports.aspx (accessed Jul 2017).

5. Frioui Mahmoudi S, Toulgui E, Ben Jeddou K, et al. Quality of life for patient with knee osteoarthritis. Ann Phys Rehabil Med 2016;59:e158-9.

6. Janssen I, Mark AE. Separate and combined influence of body mass index and waist circumference on arthritis and knee osteoarthritis. Int $J$ Obes 2006;30:1223-8.

7. Lementowski PW, Zelicof SB. Obesity and osteoarthritis. Am J Orthop 2008;37:148-51.

8. Batsis JA, Zbehlik AJ, Barre LK, et al. The impact of waist circumference on function and physical activity in older adults: longitudinal observational data from the osteoarthritis initiative. Nutr J 2014;13:81.

9. Silverwood V, Blagojevic-Bucknall M, Jinks C, et al. Current evidence on risk factors for knee osteoarthritis in older adults: a systematic review and meta-analysis. Osteoarthritis Cartilage 2015;23:507-15.

10. Loeser RF, Collins JA, Diekman BO. Ageing and the pathogenesis of osteoarthritis. Nat Rev Rheumatol 2016;12:412-20.

11. National Institutes of Health. Clinical guidelines on the identification, evaluation, and treatment of overweight and obesity in adults-the evidence report. National Institutes of Health. Obes Res 1998;6(Suppl 2):51S-209.

12. World Health Organization. Obesity: preventing and managing the global epidemic (online). 2000 file:///C:/Users/asa314/Downloads/ WHO_TRS_894\%20(2).pdf (accessed Jul 2017).

13. Riddle DL, Stratford PW. Body weight changes and corresponding changes in pain and function in persons with symptomatic knee osteoarthritis: a cohort study. Arthritis Care Res 2013;65:15-22.

14. Christensen R, Astrup A, Bliddal H. Weight loss: the treatment of choice for knee osteoarthritis? A randomized trial. Osteoarthritis Cartilage 2005;13:20-7.

15. Christensen R, Bartels EM, Astrup A, et al. Effect of weight reduction in obese patients diagnosed with knee osteoarthritis: a systematic review and meta-analysis. Ann Rheum Dis 2007;66:433-9.

16. Fernandes L, Hagen KB, Bijlsma JW, et al. EULAR recommendations for the non-pharmacological core management of hip and knee osteoarthritis. Ann Rheum Dis 2013;72:1125-35.

17. McAlindon TE, Bannuru RR, Sullivan MC, et al. OARSI guidelines for the non-surgical management of knee osteoarthritis. Osteoarthritis Cartilage 2014;22:363-88.

18. Castrogiovanni P, Musumeci G. Which is the best physical treatment for osteoarthritis? J Funct Morphol Kinesiol 2016;1:54-68

19. Abbott JH, Robertson MC, Chapple C, et al. MOA Trial team. Manual therapy, exercise therapy, or both, in addition to usual care, for osteoarthritis of the hip or knee: a randomized controlled trial. 1: clinical effectiveness. Osteoarthritis Cartilage 2013:21:525-34.

20. Foster NE, Healey EL, Holden MA, et al. BEEP trial team. A multicentre, pragmatic, parallel group, randomised controlled trial to compare the clinical and cost-effectiveness of three physiotherapyled exercise interventions for knee osteoarthritis in older adults: the BEEP trial protocol (ISRCTN: 93634563). BMC Musculoskelet Disord 2014;15:254.

21 Foster NE, Nicholls E, Holden MA, et al. Improving the effectiveness of exercise therapy for older adults with knee pain: a pragmatic randomized controlled trial. Rheumatology 2015;54(Suppl 1):i40.

22. Roddy E, Zhang W, Doherty M. Aerobic walking or strengthening exercise for osteoarthritis of the knee? A systematic review. Ann Rheum Dis 2005;64:544-8.

23. Messier SP, Loeser RF, Mitchell MN, et al. Exercise and weight loss in obese older adults with knee osteoarthritis: a preliminary study. J Am Geriatr Soc 2000;48:1062-72.

24. Messier SP, Loeser RF, Miller GD, et al. Exercise and dietary weight loss in overweight and obese older adults with knee osteoarthritis: the arthritis, diet, and activity promotion trial. Arthritis Rheum 2004:50:1501-10.

25. Messier SP, Mihalko SL, Legault C, et al. Effects of intensive diet and exercise on knee joint loads, inflammation, and clinical outcomes among overweight and obese adults with knee osteoarthritis: the IDEA randomized clinical trial. JAMA 2013;310:1263-73.

26. Skou ST, Rasmussen S, Laursen MB, et al. The efficacy of 12 weeks non-surgical treatment for patients not eligible for total knee replacement: a randomized controlled trial with 1-year follow-up. Osteoarthritis Cartilage 2015;23:1465-75.

27. Alrushud AS, Rushton AB, Kanavaki AM, et al. Effect of physical activity and dietary restriction interventions on weight loss and the musculoskeletal function of overweight and obese older adults with knee osteoarthritis: a systematic review and mixed method data synthesis. BMJ Open 2017;7:e014537.

28. Martin LR, Williams SL, Haskard KB, et al. The challenge of patient adherence. Ther Clin Risk Manag 2005;1:189.

29. Persch AC, Page SJ. Protocol development, treatment fidelity, adherence to treatment, and quality control. Am J Occup Ther 2013:67:146-53.

30. Blekken LE, Nakrem S, Gjeilo KH, et al. Feasibility, acceptability, and adherence of two educational programs for care staff concerning nursing home patients' fecal incontinence: a pilot study preceding a cluster-randomized controlled trial. Implement Sci 2015;10:72.

31. Gibbs JC, McArthur C, Milligan J, et al. Measuring the implementation of a group-based Lifestyle-integrated Functional Exercise (Mi-LiFE) intervention delivered in primary care for older adults aged 75 years or older: a pilot feasibility study protocol. Pilot Feasibility Stud 2015;1:20.

32. Devos-Comby L, Cronan T, Roesch SC. Do exercise and selfmanagement interventions benefit patients with osteoarthritis of the knee? A metaanalytic review. J Rheumatol 2006;33:744-56.

33. Ryan P, Sawin KJ. The individual and family self-management theory: background and perspectives on context, process, and outcomes. Nurs Outlook 2009;57:217-25.

34. Nilsen P. Making sense of implementation theories, models and frameworks. Implement Sci 2015;10:53.

35. Allegrante JP, Kovar PA, MacKenzie CR, et al. A walking education program for patients with osteoarthritis of the knee: theory and intervention strategies. Health Educ Q 1993;20:63-81.

36. Michie S. Designing and implementing behaviour change interventions to improve population health. $J$ Health Serv Res Policy 2008;13:64-9.

37. Cartwright D, Zander A. Group dynamics: research and theory. New York: Harper and Row, 1960.

38. National Institute for Health and Clinical Excellence. Osteoarthritis: care and management (online). $2014 \mathrm{http} / /$ guidance.nice.org.uk/ CG177 (accessed Jan 2017).

39. Bowen DJ, Kreuter M, Spring B, et al. How we design feasibility studies. Am J Prev Med 2009;36:452-7.

40. Kitzinger J. Qualitative research. Introducing focus groups. BMJ 1995;311:299-302.

41. Rabiee F. Focus-group interview and data analysis. Proc Nutr Soc 2004:63:655-60.

42. Tickle-Degnen L. Nuts and bolts of conducting feasibility studies. Am J Occup Ther 2013;67:171-6.

43. Rushton A, Heneghan NR, Heap A, et al. Patient and physiotherapist perceptions of rehabilitation following primary lumbar discectomy: a qualitative focus group study embedded within an external pilot and feasibility trial. BMJ Open 2017;7:e015878.

44. Berry PA, Jones SW, Cicuttini FM, et al. Temporal relationship between serum adipokines, biomarkers of bone and cartilage turnover, and cartilage volume loss in a population with clinical knee osteoarthritis. Arthritis Rheum 2011;63:700-7.

45. Dobson F, Hinman RS, Roos EM, et al. OARSI recommended performance-based tests to assess physical function in people diagnosed with hip or knee osteoarthritis. Osteoarthritis Cartilage 2013;21:1042-52.

46. Thiese MS. Observational and interventional study design types; an overview. Biochem Med 2014;24:199-210.

47. Lancaster GA, Dodd S, Williamson PR. Design and analysis of pilot studies: recommendations for good practice. J Eval Clin Pract 2004;10:307-12.

48. Billingham SA, Whitehead AL, Julious SA. An audit of sample sizes for pilot and feasibility trials being undertaken in the United Kingdom registered in the United Kingdom Clinical Research Network database. BMC Med Res Methodol 2013;13:104.

49. Julious SA. Sample size of 12 per group rule of thumb for a pilot study. Pharm Stat 2005;4:287-91.

50. Sim J, Lewis M. The size of a pilot study for a clinical trial should be calculated in relation to considerations of precision and efficiency. $J$ Clin Epidemiol 2012;65:301-8.

51. National Institute for Health Research. Justifying sample size for a feasibility study (online). https://www.rds-london.nihr.ac.uk/ RDSLondon/media/RDSContent/files/PDFs/Justifying-Sample-Sizefor-a-Feasibility-Study_1.pdf (accessed May 2018).

52. Eldridge SM, Chan CL, Campbell MJ, et al. CONSORT 2010 statement: extension to randomised pilot and feasibility trials. Pilot Feasibility Stud 2016;2:64.

53. Magana A. Variety in qualitative inquiry: theoretical orientations. Patton, MQ Qualitative research and evaluation methods 2002:75-143. 
54. Tong A, Sainsbury P, Craig J. Consolidated criteria for reporting qualitative research (COREQ): a 32-item checklist for interviews and focus groups. Int J Qual Health Care 2007;19:349-57.

55. Onwuegbuzie AJ, Dickinson WB, Leech NL, et al. A qualitative framework for collecting and analyzing data in focus group research. Int J Qual Methods 2009;8:1-21.

56. Krueger RA. Analyzing and reporting focus group results. London: Sage publications, 1997:6.

57. Ritchie J, Spencer L. Qualitative data analysis for applied policy research. In: bryman A, Burgess RG, eds. Analyzing qualitative data. Abingdon: Routledge, 1994:173-94.

58. Thabane L, Ma J, Chu R, et al. A tutorial on pilot studies: the what, why and how. BMC Med Res Methodol 2010;10:1.
59. Stow $\mathrm{R}$, Rushton $\mathrm{A}$, Ives $\mathrm{N}$, et al. A cluster randomised feasibility trial evaluating six-month nutritional interventions in the treatment of malnutrition in care home-dwelling adults: recruitment, data collection and protocol. Pilot Feasibility Stud 2015;1:3.

60. Stow R, Ives N, Smith C, et al. A cluster randomised feasibility trial evaluating nutritional interventions in the treatment of malnutrition in care home adult residents. Trials 2015;16:433.

61. Aitken D, Buchbinder R, Jones G, et al. Interventions to improve adherence to exercise for chronic musculoskeletal pain in adults. Aust Fam Physician 2015;44:CD005956.

62. Kanavaki AM, Rushton A, Efstathiou N, et al. Barriers and facilitators of physical activity in knee and hip osteoarthritis: a systematic review of qualitative evidence. BMJ Open 2017;7:e017042. 